\title{
Magnetic properties of the electrons in a phosphorene monolayer
}

\author{
V. V. Karpunin, N. N. Khvastunov \\ Evseviev Mordovia State Pedagogical Institute, Studencheskaya 11 A, Saransk, 340007, Russia \\ karpuninvv@mail.ru,khvastunovnn@mail.ru
}

PACS 07.55.Jg, 13.40.Em

DOI 10.17586/2220-8054-2019-10-5-536-539

\begin{abstract}
The magnetic moment of a phosphorene monolayer is calculated in the presence of a perpendicular magnetic field B. Landau levels are strongly anisotropic with different anisotropic effective masses. The oscillatory character of the magnetic moment dependences on the magnetic field is investigated.
\end{abstract}

Keywords: Magnetic moment, phosphorene monolayer.

Received: 24 September 2019

Revised: 28 September 2019

\section{Introduction}

In recent years, monolayers of black phosphorus (phosphorene) have been intensively studied. In [1], the Landau levels (LLs) and magneto-transport (MT) properties of phosphorene under a perpendicular magnetic field were studied. Owing to the anisotropic energy dispersions, i.e., the effective masses, the Landau splittings of conduction and valence band are different for a fixed magnetic field, and the wavefunctions corresponding to the LLs show strong anisotropic behavior.

In [2], the magneto-optical transport properties of a phosphorene monolayer subjected to an external perpendicular magnetic field were studied, the magneto-optical Hall and longitudinal optical conductivities as functions of temperature, a magnetic field, and Fermi energy were evaluated, and it was shown that they were strongly influenced by the magnetic field.

In [3], continuum descriptions of single layer and bilayer black phosphorus, starting from a tight binding model that reproduces the results of first-principles calculations, have been presented; also, the spectra of electrons and holes in the vicinity of the Fermi level at the $\Gamma$ point were obtained and the Landau-level spectra for both systems were calculated.

In [4], the electronic properties of $2 \mathrm{D}$ electron gas in black phosphorus multilayers due to the presence of a perpendicular magnetic field have been examined. Authors found that resonant structures in the ac conductivity exhibit a redshift with increasing doping due to interband coupling,suggesting possible electric modulation of light absorption and Faraday rotation.

In [5], the linear, the third-order nonlinear, and the total magneto-optical absorption coefficients have been studied, as well as the relative refractive index changes in monolayer phosphorene in the presence of an external perpendicular magnetic field.

In [6], the authors review recent experimental and theoretical work addressing the quantum oscillatory magnetization $M(B)$ in 2DESs subject to SOI. They first introduce a theoretical model that describes $M(B)$ by numerically solving the Hamiltonian including Rashba-SOI and Dresselhaus-SOI and the Zeeman term in an arbitrarily tilted magnetic field. Both $E_{F}(B)$ and $M(B)$ show pronounced beating patterns in low magnetic fields. This pattern is a consequence of the nonlinear LL dispersion induced by the SOI, leading to an uneven spacing of levels at $E_{F}$.

In [7], the authors investigated the optical responses of monolayer phosphorene subjected to a circular electromagnetic wave. Band structures for the monolayer phosphorene in the absence and presence of perpendicular electric field were plotted. With the aid of the tight-binding model and Kubo formalism implemented in the linear response theory, the anisotropic optical conductivity of phosphorene is being calculated. They have evaluated the reflected, transmitted and absorbed waves as a function of the electric field at optical frequencies beneath, near and above the band gap.

The microscopic mechanism of magnetization and polarization resulting from strong spin-orbital coupling of the electron gas in multiferroics was examined in [8]. Explicit analytic expressions were obtained for the magnetization and polarization of the electron gas in multiferroics.

The magnetic response of a quantum wire of elliptical cross section was investigated in [9]. An explicit analytic expression was found for the spectrum and wave functions of an electron in the wire. Using an approach based on 
classical partition function finding, an expression was obtained for the magnetic response of the electron gas in the wire. The dependence of the response on the magnitude and direction of the magnetic field was found.

The magnetic moment of single layer graphene rings is investigated in [10]. An analytical expression for the magnetic moment as a function of the magnetic field flux through the one-dimensional quantum rings was obtained. This expression has the oscillation character. The oscillation period is equal to one flux quanta.

The absorption coefficient of the electromagnetic radiation in a phosphorene single layer placed in a magnetic field was found in [11]. A degenerate and nondegenerate electron gas is being considered. The resonant dependences of the absorptance on the radiation frequency and applied magnetic field were found.

Let us consider a quasi-one-dimensional electron gas in a phosphorene monolayer. An analytical expression for the LLs in low energy regime is obtained via solving the decoupled Hamiltonian, which agrees well with the numerical calculations $[1,2]$. The Hamiltonian of such a system is defined by the expression $[1,2]$ :

$$
H=\left(\begin{array}{cc}
E_{c}+\left(\alpha^{\prime} \Pi_{x}^{2}+\beta \Pi_{y}^{2}\right) / 2 & 0 \\
0 & E_{v}-\left(\lambda^{\prime} \Pi_{x}^{2}-\eta \Pi_{y}^{2}\right) / 2
\end{array}\right),
$$

where $E_{c}$ corresponds to the boundary of the conduction band, $E_{v}$ corresponds to the boundary of the valence band, $\alpha^{\prime}=\alpha+\gamma^{2} / E_{g}, \lambda^{\prime}=\lambda+\gamma^{2} / E_{g}, \gamma=8.5 \cdot 10^{5} \mathrm{~m} / \mathrm{s}, \alpha=1 / m_{c x}, \beta=1 / m_{c y}, \lambda=1 / m_{v x}, \eta=1 / m_{v y}$, $m_{c x}=0.793 m_{e}, m_{c y}=0.848 m_{e}, m_{v x}=0.1 .363 m_{e}, m_{v y}=1.142 m_{e}, m_{e}$ is the free electron mass, $E_{g}=$ $E_{c}-E_{v}=1.52 \mathrm{eV}, \boldsymbol{\Pi}=\mathbf{p}+\frac{e}{c} \mathbf{A}$ is a generalized momentum operator.

The energy spectrum of the eigenstates of electrons in a magnetic field can be written as follows [1,2]:

$$
E_{n, s}=E_{s}+s\left(n+\frac{1}{2}\right) \hbar \omega_{s}
$$

where $s \pm 1$ corresponds to the boundary of the conduction and valence band, formula universally describes the Landau levels of the conduction band and the valence band, $E_{s}=E_{+}=E_{c}$ for conduction band, $n=0,1,2,3 \ldots$, $\omega_{+}=\omega_{c}^{\prime}=e B /\left(m_{c x}^{\prime} m_{c y}\right)^{1 / 2} c=2.657 \omega_{e}$ is modified cyclotron frequency, $m_{c x}^{\prime}=0.167 m_{e}, \omega_{e}=e B / m_{e} c$.

The article is organized as follows: The magnetic moment is being calculated by two methods and the results are being discussed.

\section{Magnetic moment}

The classical expression of the thermodynamic potential has the form [13]:

$$
\Omega=-T \sum_{n=0}^{\infty} \ln \left(1+\exp \left(\frac{\mu-E_{n,+}}{T}\right)\right),
$$

here $\mu_{B}$ is the Bohr magneton, $\mu$ is chemical potential. Then for the magnetic moment we get:

$$
-M=\sum_{n=0}^{\infty} \frac{d E_{n,+}}{d B}\left(1+\exp \left(\frac{E_{n,+}-\mu}{T}\right)\right)^{-1}
$$

where

$$
\frac{d E_{n,+}}{d B}=a\left(n+\frac{1}{2}\right) \mu_{B}
$$

where $a=5.314$. Finally we can obtain:

$$
-\frac{\left.M(T)\right|_{T \neq 0}}{\mu_{B}}=\sum_{n=0}^{\infty} a\left(n+\frac{1}{2}\right) \frac{1}{1+\exp \left(E_{n,+}-\mu\right) / T} .
$$

In the second method thermodynamic potential $\Omega$ can be obtained from the dependence $\Omega(Z)[8,10]$. This method is applied in [12] for three-dimensional electron gas Then we obtain:

$$
\Omega=\frac{E_{+}}{2 \pi i} \int_{\alpha-i \infty}^{\alpha+i \infty} \frac{Z(\xi) d \xi}{\xi^{2}} \int_{0}^{\infty} \exp (E \xi) \frac{\partial f_{0}}{\partial E} d E
$$

where $f_{0}(E)$ is the Fermi distribution function and $\alpha \leq 1 / T$.

The statistical sum has the form:

$$
Z(\xi)=\sum_{n=0}^{\infty} \exp \left(-\xi \varepsilon_{n}\right), \quad \xi=E_{+} / T
$$


We use the approach $[8,10]$ in order to find $\Omega$. Let us introduce the notation $z\left(\varepsilon_{F}\right)$ :

$$
z(\varepsilon)=\frac{1}{2 \pi i} \int_{\alpha-i \infty}^{\alpha+i \infty} \frac{\exp (\varepsilon \xi) Z(\xi) d \xi}{\xi^{2}} .
$$

As is clear from (7) $\Omega_{0}=\Omega(T=0)=-z\left(\varepsilon_{F}\right) E_{0}$, where $\varepsilon_{F}=E_{F} / E_{+}$and $E_{F}$ is Fermi energy. We can represent (9) in the form:

$$
z\left(\varepsilon_{F}\right)=\frac{1}{2 \pi i} \int_{\alpha-i \infty}^{\alpha+i \infty} \sum_{n=0}^{\infty} \exp \left[\left(\varepsilon_{F}-\varepsilon_{n}\right) \xi\right] \frac{d \xi}{\xi^{2}} .
$$

We rewrite magnetic moment of the phosphorene monolayer $\mathbf{M}=-\partial \Omega / \partial \mathbf{B}$ in the convenient form:

$$
M=-\frac{\partial \omega_{+}}{\partial B} \frac{\partial \Omega}{\partial \omega_{+}}=-2.657 \frac{e}{m_{0} c} \frac{\partial \Omega}{\partial \omega_{+}} .
$$

It should be noted that from the formula (9) one must get the expression for $\partial z / \partial \omega_{+}$:

$$
\frac{d z(\varepsilon)}{d \omega_{+}}=\frac{1}{2 \pi i} \int_{\alpha-i \infty}^{\alpha+i \infty} \exp (\varepsilon \xi) \frac{1}{\xi^{2}} \frac{d Z}{d \omega_{+}} d \xi,
$$

where

$$
\frac{d Z}{d \omega_{+}}=-\xi \sum_{n=0}^{\infty} \exp \left(-\varepsilon_{n} \xi\right) \frac{\hbar(n+1 / 2)}{E_{+}} .
$$

As it follows from (13),(12) $d z / d \omega_{+}$has the form:

$$
\frac{d z(\varepsilon)}{d \omega_{+}}=-\frac{1}{2 \pi i} \int_{\alpha-i \infty}^{\alpha+i \infty} \sum_{n=0}^{\infty} \frac{\exp \left(\left(\varepsilon-\varepsilon_{n}\right) \xi\right)}{\xi} \frac{\hbar(n+1 / 2)}{E_{+}} d \xi .
$$

To take the integral in (14), we need to use [14]:

$$
\frac{1}{2 \pi i} \int_{\alpha-i \infty}^{\alpha+i \infty} z^{\gamma-1} e^{-p z} d z= \begin{cases}0, & p>0 \\ \frac{1}{(-p)^{\gamma} \Gamma(1-\gamma)}, & p<0 .\end{cases}
$$

In the case $T \neq 0$ we obtain from (11):

$$
-\frac{\left.M(T)\right|_{T \neq 0}}{\mu_{B}}=\sum_{n=0}^{\infty} a\left(n+\frac{1}{2}\right) \frac{1}{1+\exp \left(E_{n,+}-\mu\right) / T},
$$

here $\mu_{B}$ is the Bohr magneton, $\mu$ is chemical potential, $m_{0}$ is the free electron mass, $a=5.314$.

\section{Conclusion}

The expression of the magnetic moment of phosphorus electrons was obtained by two methods. The first method is to calculate the classical expression of the thermodynamic potential and then obtain the magnetic moment; the second method is to calculate the thermodynamic potential through the contour integral and then obtain the magnetic moment. Both methods leads to the same expression for the magnetic moment. Note that the second method also makes it easy to go to the $T \rightarrow 0$ case. The derivative of the Fermi distribution function is equal to the Dirac delta function. This makes it easy to take the second integral in (7).

Figure 1 shows the oscillatory dependence of the magnetic moment on the inverse magnetic field. Oscillation peaks are smoothed by temperature. The effect should be observed at high magnetic fields, oscillation attenuation occurs in weak fields. This corresponds to de Haas van Alphen oscillations. It should be noted that similar oscillations were also observed in other low-dimensional structures [15]. 


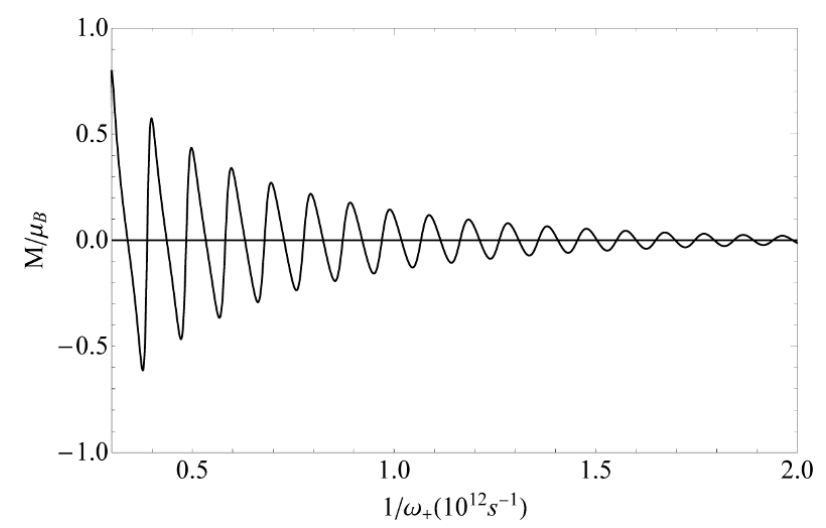

FIG. 1. Dependence of the magnetic moment on the reverse magnetic field, $\mu / T=1000, s=+1$

\section{References}

[1] Zhou X.Y., Zhang R., et al. Landau levels and magneto-transport property of monolayer phosphorene. Sci. Rep., $2015, \mathbf{5}, 12295$.

[2] Tahir M., Vasilopoulos P., Peeters F.M. Magneto-optical transport properties of monolayer phosphorene. Phys. Rev. B, 2015, 92, 045420.

[3] Pereira J.M.Jr., Katsnelson M.I. Landau levels of single-layer and bilayer phosphorene. Phys. Rev. B, $2015,92,075437$.

[4] Jiang Y., Roldan R., Guinea F., Low T. Magnetoelectronic properties of multilayer black phosphorus. Phys. Rev. B, $2015,92,085408$.

[5] Nguyen C.V., Hieu N.N., et al. Linear and nonlinear magneto-optical properties of monolayer phosphorene. J. Appl. Phys., $2017,121,045107$.

[6] Wilde M.A., Rupprecht B., et al. Spinorbit interaction in the magnetization of two-dimensional electron systems. Phys. Status Solidi B, 2014, 251, P. 1710-1724.

[7] Lea P.T.T., Yarmohammadi M. Perpendicular electric field effects on the propagation of electromagnetic waves through the monolayer phosphorene. J. Magn. Magn. Mater., 2019, 491, 165629.

[8] Margulis V.A. Magnetization and polarization of the electron gas in multiferroics. Low Temp. Phys., 2014 40, P. 363-366.

[9] Margulis V.A., Karpunin V.V., Mironova K.I. Magnetic response of a quantum wire of elliptical cross-section in a magnetic field perpendicular to the axis of the wire. Nanosystems: Phys. Chem. Math., 2018, 9 (2), P. 244-251.

[10] Margulis V.A., Karpunin V.V., Mironova K.I. Magnetic moment of single layer graphene rings. Sol. St. Commun., 2018, 269, P. 108-111.

[11] Margulis V.A., Karpunin V.V. Resonance Absorption of Electromagnetic Radiation in a Phosphorene Single Layer. Semiconductors, 2019, 53, P. 458-464.

[12] Rumer Yu.B. On the theory of electron gas magnetism. Zh. Eksp. Teor. Fiz., 1948, 18, P. 1081-1095.

[13] Landau L.D., Lifshitz E.M. Statistical Physics, Part 1, 3rd ed., Pergamon Press, Oxford, 1980, 544 p.

[14] Prudnikov A.P., Brychkov Yu.A., Marichev O.I. Integrals and series, Elementary Functions, N. Y.: Gordon and Breach, 1986,808 p.

[15] Zhang S., Ma N., Zhang E. The modulation of the de Haasvan Alphen effect in graphene by electric field. J. Phys.: Condens. Matter, 2010, 22, 115302. 